\title{
Misión Sucre: Perfil estudiantil y opiniones de sus actores directos en Maracaibo*
}

\author{
Peña Ruiz, Rosa Mercedes** \\ Parra Olivares, Javier Enrique*** \\ Méndez de Souki, Betty****
}

\section{Resumen}

Desde el año 2003 el Estado venezolano viene trazando diferentes estrategias en la búsqueda de potenciar la sinergia institucional y la participación comunitaria para garantizar el acceso a la educación universitaria a todos los bachilleres sin cupo y superar su condición de excluidos de ese subsistema de educación. En ese marco se definió la Misión Sucre. El presente artículo examina dos aspectos de la misma: a) el perfil de la población estudiantil incorporada y b) la opinión que sobre la ejecución de la Misión Sucre en el municipio Maracaibo tienen sus actores más inmediatos (estudiantes, profesores y coordinadores de aldeas universitarias). Para ello se revisaron documentos oficiales y se aplicaron cuestionarios auto-administrados y entrevistas semi-estructuradas a una muestra probabilística de los actores. Se concluye que la Misión Sucre, en gran parte, ha cumplido el objetivo de incorporar a los bachilleres que anteriormente no habían sido admitidos en la educación universitaria, y que los actores involucrados opinan que se han logrado implementar nuevos modelos educativos.

Palabras clave: Misión Sucre, Perfil estudiantil, Opinión de actores, Políticas públicas universitarias.

Recibido: 10-07-09. Aceptado: 05-11-09

* Investigación financiada por el Consejo de Desarrollo Científico y Humanístico de la Universidad del Zulia (CONDES).

** Socióloga, Magíster Scientarium en Gerencia Pública. Profesora de la Universidad Bolivariana de Venezuela. Estudiante del Doctorado en Ciencias para el Desarrollo Estratégico en el área de conocimiento Gestión de políticas públicas, dictado por la Universidad Bolivariana de Venezuela.

*** Sociólogo, Especialista en Teoría Sociológica y en Análisis de Datos. Profesor de la Universidad del Zulia. Doctor en Ciencias Sociales mención Gerencia.

**** Licenciada en Educación, Magíster en Educación. Profesora jubilada de la Universidad del Zulia. 


\title{
The Sucre Mission: Student Profile and Opinions from its Direct Actors in Maracaibo
}

\begin{abstract}
Since 2003, the Venezuelan State has been following different strategies in the search for empowering institutional synergy and community participation to guarantee access to university education for all high-school graduates without a place in the university and overcome the condition of exclusion in this education subsystem. The Sucre Mission was defined within this framework. This article examines two aspects of it: a) a profile for the incorporated student population and b) the opinion the most immediate actors (students, professors and coordinators of village universities) have regarding execution of the Sucre Mission in the municipality of Maracaibo. To accomplish this, official documents were reviewed and self-administered questionnaires and semi-structured interviews were applied to a random sampling of the actors. Conclusions were that the Sucre Mission, to a great extent, has fulfilled its objective of incorporating high school graduates who previously had not been admitted to university education and that the actors involved are of the opinion that new educational models have been implemented.
\end{abstract}

Key words: Sucre Mission, student profile, actor's opinion, university public policies.

\section{Introducción}

En los dos últimos períodos del gobierno democrático representativo, el Estado fue reduciendo gradualmente sus compromisos en materia educativa y específicamente en el sector de la educación universitaria, lo que se evidencia en una inversión descendente desde el año 1989 a 1998, afectada por el interés en realizar recortes presupuestarios en todas las políticas sociales. De igual manera, se fueron implementando mecanismos de ingreso soportados en los promedios de notas de los bachilleres en educación media y diversificada y en la prueba de aptitud académica a lo que, sucesivamente, se le incorporan nuevos criterios en cada universidad y algunas carreras especificas hasta llegar al año 2000 , cuando las denominadas "políticas" se reducen a una selección cada vez más restrictiva de los ingresos de los estudiantes a la educación universitaria.

Esto generó una amplia deuda social acumulada, pues la matrícula universitaria sufrió un estancamiento que favoreció la exclusión de los estudiantes provenientes de los sectores más pobres. Diversas investigaciones (Morales Gil, 2003 y Fuenmayor y Vidal, 2000) señalan que la incorporación a la educación universitaria benefició a los sectores de mayores recursos, a la población de las grandes ciudades y a los estudiantes procedentes de planteles privados. A la par de este fenómeno, la educación universitaria oficial redujo notablemente su influencia en favor de la de gestión privada.

Los estudios anteriores señalan que los estudiantes procedentes de sectores de clase media y alta tenían mejo- 
Misión Sucre: Perfil estudiantil y opiniones de sus actores directos en Maracaibo Peña Ruiz, Rosa M.; Parra Olivares, Javier E. y Méndez de Souki, Betty

res promedios en bachillerato y en la puntuación de los resultados de la prueba de aptitud académica. Estas diferencias no estaban relacionadas con su asignación a las universidades, ya que en 1984 eran mayores los porcentajes de inclusión de jóvenes de los sectores más bajos en la estratificación social, y es en 1998 cuando las injusticias se hacen más evidentes. Para ilustrar este punto, se puede constatar que en 1984 el 70,9\% de la demanda de ingreso de los estratos más bajos era cubierta por las universidades públicas, mientras que en 1998 sólo era cubierto el $19,7 \%$ de la demanda de esos estratos. Por su parte el estrato alto pasó de 55\% de cobertura de su demanda de ingreso en 1984 a $99,9 \%$ en 1998. (Fuenmayor y Vidal, 2000).

Estas cifras brindan una clara panorámica de cómo las universidades públicas fueron reduciendo su capacidad de ingreso para los sectores más pobres de la sociedad.

Por otro lado, Fuenmayor y Vidal (2000) demuestran que, entre $1984 \mathrm{y}$ 1998, se desarrollaron transformaciones en los mecanismos de admisión existentes en el país, trasladándose la decisión del Consejo Nacional de Universidades a manos de los procesos internos en cada universidad. Estos elementos se suman a la situación ya planteada anteriormente.

Otros resultados de los mismos estudios realizados por Fuenmayor y Vidal (2000) revelan cómo evolucionó el ingreso al sistema de educación universitaria entre los años 1984 y 1998. Sus datos muestran que la prueba de aptitud académica y los mecanismos internos de las universidades aparecen claramente como responsables de una selección muy desigual, evidenciándose que los resultados tienden a favorecer a los estudiantes de mayores recursos socioeconómicos; situación que es similar para los mecanismos de selección interna de las universidades, siendo más acentuada en este último caso. Así mismo, constatan un descenso desde 1984 hasta 1998 en los cupos para los bachilleres que egresaban del sector público de la educación. El sector oficial perdió un poco más de una tercera parte de los cupos en términos porcentuales. Paralelamente, los egresados del sector privado ocuparon casi diez por ciento más.

Tomando en cuenta los datos anteriores, podría afirmarse que las instituciones universitarias, a través de sus propios mecanismos, permitieron que, entre el año 1984 y 1998, se cambiara un patrón paritario de ingreso por condición socioeconómica o por plantel de procedencia, hacia un sistema injusto que privilegia a los estratos alto y medio-alto y a los egresados de planteles privados. Esta distorsión aparece claramente asociada a la generalización de los mecanismos internos de ingreso.

Se aprecia así que el problema de inequidad en el acceso a la educación universitaria está relacionado con la profundización de las desigualdades sociales, las cuales generan exclusión social y educacional en los niveles precedentes, agudizándose a medida que se asciende en los distintos niveles del sistema escolar.

Para abordar esta realidad, desde 1999 el Gobierno Nacional ha diseñado políticas y estrategias de acción tendientes a subsanar la exclusión social y educacional extendida y agudizada en déca- 
das anteriores, especialmente en aquellos sectores poblacionales de bajos recursos socio-económicos, a propósito de insertarlos en la educación universitaria, de acuerdo a lo planteado en la Constitución (esto es: sin más limitaciones que las que se derivan de sus aptitudes, vocación y aspiraciones).

En este marco se ubica la Misión Sucre, como política de Estado que plantea un impulso a la educación gratuita, obligatoria y de calidad, cuya población objetivo está conformada por quienes han sido excluidos por las políticas del sistema escolar venezolano a nivel universitario. Surge en el año 2003 como una iniciativa del Gobierno Nacional para potenciar la sinergia institucional y la participación comunitaria, para superar la exclusión en la educación universitaria (Ministerio de Educación Superior, 2003).

Una de las directrices más importantes de la Misión Sucre es la de encontrar cabida a todos en el sistema de educación universitaria, a través de la creación de las aldeas universitarias, que son espacios que se instalan en cada municipio del territorio venezolano, en los cuales trabajan diferentes instituciones de educación universitaria con distintas carreras (programas) que se estimen deben impartirse en cada uno de los municipios, lo cual ha sido denominado como municipalización de la educación superior.

La presente investigación indagó a) el perfil de los(as) bachilleres incorporados(as) a la Misión Sucre en Maracaibo y b) las opiniones que, sobre la ejecución de esta política educativa tienen los actores involucrados directamente en la misma (coordinadores de aldeas universitarias, profesores y estudiantes), como par- te de una evaluación mayor de los resultados de esta política educativa.

La investigación fue de tipo descriptivo, a partir de documentos oficiales y el uso de un cuestionario autoadministrado para estudiantes y profesores, así como una entrevista semiestructurada aplicada a los coordinadores de las aldeas universitarias del municipio Maracaibo.

Se definieron dos universos: el integrado por todos los estudiantes y el conformado por todos los profesores y coordinadores de la Misión Sucre que participan en las aldeas universitarias del municipio Maracaibo. Se delimitaron tantas poblaciones como indicadores contenidos en los instrumentos de recolección de datos, con base a la definición de población estadística como el conjunto integrado por todas las mediciones de un indicador o variable en un universo definido (Parra Olivares, 2006).

El diseño de muestreo probabilístico empleado fue el aleatorio por conglomerados en dos etapas (bi-etápico). Los conglomerados de primera etapa son las 22 aldeas universitarias que funcionaban en el municipio Maracaibo para el Ministerio de Educación Superior hasta mayo de 2008. De éstas se escogieron al azar siete, a saber: Liceo Rafael María Baralt, Ince Marrón, Liceo Francisco José Duarte, Unidad Educativa Manuel Segundo Sánchez, Colegio Cosme González, Unidad Educativa Raúl Leoni e Instituto Universitario de Tecnología de Maracaibo. Los conglomerados de segunda etapa fueron los salones que conforman las 7 aldeas seleccionadas al azar.

El tamaño de muestra calculado inicialmente fue de 304 estudiantes, con un error máximo admisible de $4,5 \%$ para 
Misión Sucre: Perfil estudiantil y opiniones de sus actores directos en Maracaibo Peña Ruiz, Rosa M.; Parra Olivares, Javier E. y Méndez de Souki, Betty

todas las variables cualitativas, un nivel de confianza del $95 \%$ y como varianza de indicador clave se usó 0,16 , asumiendo la variabilidad del indicador "Tiempo de ingreso del estudiante a la Misión Sucre" considerado clave en el estudio. Para este indicador se obtuvo una varianza [p(1-p)] de 0,16 en la muestra piloto, por cuanto las proporciones de alumnos fueron de 0,2 para los alumnos con menos de un año en la Misión Sucre y 0,8 para los estudiantes con un año o más.

Así mismo, la muestra piloto permitió estimar que en cada salón de las aldeas de Maracaibo hay un promedio de 21,7 estudiantes. Es decir que para poder acceder a un total igual o mayor a 304 estudiantes se requería seleccionar 14 salones en las aldeas escogidas aleatoriamente. En definitiva, se decidió tomar entonces 2 salones al azar en cada una de las 7 aldeas de la muestra. Es de resaltar que, al finalizar el censo en los 14 salones elegidos (unidades de muestreo de segunda etapa) en las 7 aldeas seleccionadas (unidades de muestreo de primera etapa), el total de estudiantes incluidos en la muestra fue de 315. Por su parte, el total de profesores que impartían clase el día de toma de los datos en los 14 salones fue de 39 , en diferentes unidades curriculares.

\section{Políticas Públicas y Dimensión Educativa}

Las políticas públicas representan un proceso continuo de toma de decisiones del Estado para la resolución de problemas de diferente índole. No son acciones imparciales desprovistas de contenido ideológico ni son el resultado de deci- siones unilaterales. Por el contrario, constituyen una forma particular de gestión del Estado para enfrentar los desafíos del desarrollo social. En este sentido las políticas públicas forman parte del quehacer del Estado, desde la ejecución de planes hasta la omisión de los mismos ante las demandas de la sociedad.

De cualquier manera es posible establecer que "lo político" y "la política" se refieren a las acciones de un determinado gobierno, orientadas a atender determinados fines y dirigidas a una población. Es decir, un medio para llegar a una determinada meta social.

De acuerdo a lo planteado, conviene discutir ahora algunos conceptos de políticas públicas manejados por diferentes autores.

Subirats (1996) señala que se entiende por política pública al instrumento de trabajo a través del cual se pretenden alcanzar desde el Estado, de manera sistemática y coherente, ciertos objetivos de interés para el bienestar de toda la sociedad. Asimismo, plantea que las políticas públicas son procesos, decisiones y resultados, pero sin que ello excluya conflictos entre intereses presentes en cada momento, tensiones entre diferentes definiciones del problema a resolver y entre diferentes racionalidades organizativas y de acción.

Similarmente, Valles (2000) establece que una política pública puede ser concebida como un conjunto de decisiones, que tienen como foco una determinada área de conflicto o tensión social. Estas decisiones son asumidas formalmente en el marco de las instituciones públicas lo cual le confiere la capacidad de obligar, pero han sido precedidas de un 
proceso de elaboración en el cual han participado diversos actores públicos y privados. Es decir, una política pública representa una fusión de decisiones y no decisiones que involucran a diferentes actores con la finalidad de llegar a establecer un acuerdo mínimo en torno a los medios más adecuados para resolver el problema o conflicto suscitado.

Una de las fases más controversiales de la formación de políticas públicas es el proceso de toma de decisiones debido a que éste involucra diferentes intereses, valores y relaciones de poder entre los actores que entran en juego. A este respecto, la toma de decisiones resulta de un proceso complejo de negociación de los actores que tratan de llegar a establecer un acuerdo mínimo en torno a los medios más adecuados para resolver el problema o conflicto ocasionado, para lo cual se considera como condición previa obtener la mayor cantidad de información posible que le permita prever las consecuencias que determinada decisión pueda tener.

No obstante, es importante tener en cuenta que en todo proceso de toma de decisión los individuos o grupos involucrados poseen distintos niveles de poder, recursos y protagonismo y, por tanto, distintos grados de influencia sobre las decisiones, por lo que la distribución del poder se plantea como un factor clave para entender sus efectos y posibles consecuencias en la formación de las políticas públicas. En este sentido, siguiendo a Subirats (1989), el proceso de toma de decisiones alude a la definición de objetivos y la fijación de las opciones o vías de acción a seguir, caracterizándose por un constante fluir de cooperación y conflicto, en una especie de mercado político, en el que precisamente, la competencia abierta asegura el respeto democrático en la determinación de las opciones.

En referencia a cómo se llevan a cabo las políticas publicas, Álvarez (1994:305) señala que son el reflejo de la “...distribución regularmente desigual del poder en la sociedad. El modo en que el poder está distribuido afecta el proceso de formación de políticas en todas sus fases...". En este sentido, se asume que las políticas públicas atienden a todo un proceso, en el que interactúan, mediante el poder que dispongan, los diferentes actores tanto públicos como privados en el impulso o limitaciones a la formulación, implementación y evaluación de aquéllas. En síntesis, se puede considerar a las políticas públicas como una línea de acción decidida por las autoridades gubernamentales y dirigidas a satisfacer fines y/o preferencias, mediante acciones que son llevadas a cabo por una red de actores públicos y privados e implica movilización de recursos; este proceso está orientado a obtener resultados en un tiempo determinado.

Es pertinente destacar que esta investigación se apoyó en el concepto que define Ángel Álvarez (1992), quien afirma que una política pública es el conjunto de acciones o actividades de las instituciones de gobierno que actúan directamente o a través de agentes y que va dirigido a tener una influencia determinada sobre la vida de los ciudadanos.

El anterior conjunto de conceptos y sus relaciones se emplearon para examinar las opiniones que tienen los actores sobre la Misión Sucre.

Al respecto es pertinente retomar a Álvarez (1994), quien plantea que en la im- 
Misión Sucre: Perfil estudiantil y opiniones de sus actores directos en Maracaibo Peña Ruiz, Rosa M.; Parra Olivares, Javier E. y Méndez de Souki, Betty

plementación al igual que en la formulación, también interactúan diversas personas y grupos, mediante el poder de que dispongan, para orientar la implementación de una determinada política de acuerdo a su conveniencia. Incluso no debe extrañar que grupos opositores o que han tenido que ceder cuotas de poder, en la fase de formulación, tengan luego privilegio en la implementación de la política.

No obstante, es en esta fase del proceso donde se ejecutan las acciones para alcanzar los objetivos que fueron planteados en la fase de formulación de la política. Ahora bien, existe una cantidad de esfuerzos públicos y privados para hacer posible que los objetivos señalados en la formulación sean viables a la hora de la implementación de la política. En ese orden de ideas Valles (2000:385) plantea: “...La implementación de políticas públicas puede ser presentada como una operación de rutina y sin demasiado interés. Las administraciones aplicarían dócilmente las decisiones adoptadas por las instituciones políticas: leyes, normas dictadas por el ejecutivo. Pero un análisis de la realidad nos hace ver que las cosas no son así. Políticas aparentemente bien diseñadas y adoptadas con apoyos mayoritarios no obtienen los resultados previstos".

Este enfoque conceptual orienta la presente investigación sobre la Misión Sucre, definida como política educativa alternativa del Estado venezolano.

La política educativa forma parte de un proyecto social amplio; por ende, debe pensarse en una articulación íntima dialéctica con la planificación global que la sociedad construye como su proyecto, y que se realiza por medio de la acción del Estado (Schneckenberg, 2003). En ella subyace una intencionalidad de cambio o de innovación, en este sentido las transformaciones educativas se presentan como elementos fundamentales en la elaboración de las propuestas.

De acuerdo con Schneckenberg (2003) dos aspectos caracterizan a la política educativa: a) actúa sobre la educación pero no tiene el dominio absoluto sobre ella pues, como práctica social, la educación sobrepasa a la escuela y, en consecuencia, incide en ella, ya que su implementación depende de las relaciones establecidas en la práctica y b) proyecta la formación de las personas que la sociedad desea perfilar. Sus objetivos son los que definirán qué tipo de individuo y de sociedad se está buscando. De igual manera la educación, para fines determinados, "...está cargada de intenciones, y son justamente las intenciones lo que tienen en común todos los tipos de política educativa" (Schneckenberg, 2003: 4).

Tomando en cuenta lo anterior y lo definido como políticas publicas en los puntos previos, se percibe entonces que la educación forma parte de un proceso social que debe ser objeto de atención por parte del Estado que planifica y proyecta en pro de los intereses globales.

\section{Resultados de la investigación}

Este aparte presenta la descripción y análisis de los resultados del estudio en cuanto a 1) el perfil del estudiante incorporado a la Misión Sucre en el municipio Maracaibo y 2) las opiniones acerca del desarrollo o puesta en marcha de la Mi- 
sión Sucre desde la perspectiva de los actores involucrados en su ejecución (profesores, estudiantes y coordinadores de aldeas), con base al cumplimiento de los objetivos y las acciones realizadas. La Misión Sucre se plantea dos objetivos generales, a saber:

“1) Facilitar la incorporación y prosecución de estudios en la educación universitaria de todos los bachilleres y bachilleras que, a pesar de sus legítimas aspiraciones y plenos derechos, no han sido admitidos o admitidas en ninguna institución de educación universitaria oficial, e incrementar el nivel educativo de la población venezolana y 2) Promover la reflexión, discusión, concepción e implantación de nuevos modelos educativos universitarios, con base en los imperativos de la democracia participativa y protagónica, el diálogo con los actores involucrados teniendo como referencia fundamental el proceso histórico, social y político que vivimos" (Ministerio de Educación Superior, 2003).

3.1. Perfil del estudiante incorporado a la educación universitaria a través de la Misión Sucre en el municipio Maracaibo

Uno de los principales objetivos de la Misión Sucre es facilitar la incorporación en el sistema de educación universitaria de las personas que, por diversos factores, se encontraban fuera de ella. En el caso del Estado Zulia, desde el año 2003 hasta la actualidad, han ingresado 48397 estudiantes a la Misión Sucre y de ellos 12369 en el municipio Maracaibo (Fundación Misión Sucre, 2009).
El perfil del estudiante se asume como la caracterización del estudiantado en relación a ciertos indicadores sociodemográficos. En este caso, el perfil de la Misión Sucre en Maracaibo se evidencia a través de los siguientes resultados obtenidos en la investigación:

\section{a) Estrato social:}

Los datos arrojaron que el $62,5 \%$ de los estudiantes pertenecen al estrato IV (pobreza relativa) de acuerdo con la técnica de Graffar (CORPOZULIA-FUNDACREDESA, 2001) y un $25,4 \%$ pertenecen a los estratos de II y III (medio-alto y medio-bajo); lo que muestra que en el caso de Maracaibo la Misión Sucre ha contribuido a incrementar la cobertura en las instituciones de educación universitaria, aún en sectores de la población con mayores posibilidades económicas. El porcentaje tan bajo $(12,1 \%)$ de estudiantes de estrato $\mathrm{V}$ (pobreza extrema), podría explicarse por la exclusión que se generó también en la educación media, por lo cual los excluidos de este estrato apenas ahora comienzan a demandar ingreso a la educación universitaria, después de cursar en la Misión Ribas.

Este resultado se corresponde con las tendencias reportadas a nivel nacional por varios estudios, en el sentido de la desigualdad de oportunidades para acceder a la educación universitaria, la cual ha sido una de las críticas más severas a la gestión educativa en el país. El mencionado acceso guarda relación con la condición social de las familias de los estudiantes que solicitan incorporarse en las instituciones de este nivel. Esto se observa en las estadísticas de la OPSU (2002) que reflejan que, a pesar del crecimiento acelerado de la matricula estudiantil en la 
Misión Sucre: Perfil estudiantil y opiniones de sus actores directos en Maracaibo Peña Ruiz, Rosa M.; Parra Olivares, Javier E. y Méndez de Souki, Betty

última mitad del siglo $\mathrm{XX}$, existe un alto porcentaje de estudiantes que no pudieron ingresar en las instituciones de educación universitaria siendo, fundamentalmente, los de los estratos más bajos los más excluidos.

El estudio de Morales (2003) que analizó la etapa de 20 años entre 1981 y el 2000 , mostró que se produjo un proceso regresivo en el cual los círculos minoritarios y privilegiados de los estratos I y II (los más elevados en la estratificación tipo Graffar) de la sociedad venezolana, incrementaron sustancialmente su participación en la matrícula de las universidades financiadas por el Estado, en deterioro de los restantes sectores sociales, fundamentalmente de las clases sociales en situación de pobreza relativa y de pobreza extrema.

En el mismo lapso los estudiantes procedentes de los institutos privados de educación media aumentaron de manera progresiva y sistemática su participación en la matrícula de las Universidades oficiales, desplazando a los alumnos que provienen de los planteles del sector público.

Según el estudio de Morales en Venezuela los estratos I y II han incrementado sustancialmente su participación en la matrícula de las universidades públicas.

b) Liceo de procedencia y tiempo de graduado(a) de bachiller.

Los estudios realizados por la Oficina de Planificación del Sector Universitario (OPSU) mostraron que el proceso de admisión a través de la prueba nacional favorecía cada vez más a estudiantes con mejores condiciones sociales. Esta situación ha sido atribuida a que los planteles de mejor calidad educativa están ubicados en el sector privado. Los estu- diantes que pueden pagar la educación privada en los establecimientos más costosos con mejores recursos escolares, son los que más oportunidades tienen de salir bien en el examen de selección por las características culturales de la prueba (Sarco Lira y Bonucci, 1999).

Fuenmayor y Vidal (2000) reportan que en las décadas de los ochenta y los noventa, el sistema de educación universitaria continuó creciendo y al comparar los años 1984 y 1998, observan un incremento en la demanda del $55,4 \%$. Sin embargo destacan como este incremento no resulta homogéneo respecto a la proveniencia de los aspirantes de colegios del sector público y privado ya que el porcentaje de incremento de los aspirantes provenientes de planteles privados en el lapso es de $134,5 \%$ (26.222 aspirantes en el 84 y 62.426 en el 98 ).

En el caso de nuestro estudio, los resultados muestran que el mayor porcentaje $(75,9 \%)$ de los estudiantes que cursan sus estudios universitarios por vía de la Misión Sucre provienen de liceos públicos y un $24,1 \%$ provienen de liceos privados. Esto coincide con las cifras anteriores que demuestran que la gran mayoría de los estudiantes que fueron excluidos para la época venían de realizar sus estudios de secundaria en liceos públicos.

Lo anterior demuestra que la Misión Sucre ha contribuido a incorporar a la educación universitaria a aquellos estudiantes que fueron excluidos en años anteriores. Esto lo podríamos reforzar aún más, cuando se cruza el tipo de liceo donde obtuvo su titulo de bachiller con el estrato social al que pertenece el estudiante, obteniendo como resultado que la gran mayoría de los estudiantes del es- 
trato $\mathrm{V}$ (pobreza extrema) egresaron de liceos oficiales $(84,2 \%)$ seguido del estrato IV (pobreza relativa) con $76,6 \%$.

c) Distribución por género y grupos de edades

El mayor porcentaje de estudiantes que hasta la actualidad ha ingresado en la Misión Sucre es del género femenino $(67 \%)$. Este resultado coincide con la tendencia de los datos tomados del censo nacional que elaboró la Misión Sucre, en el cual el género femenino representa un $63 \%$ de los estudiantes (Fundación Misión Sucre, 2003).

\subsection{Opiniones de los actores involucrados con relación a los nuevos modelos educativos universitarios en la Misión Sucre}

En esta época en que los conocimientos científicos y tecnológicos se desarrollan tan rápida y ampliamente, determinando el surgimiento de nuevas disciplinas o la reestructuración interna de ellas, la educación universitaria, como generadora de este dinámico proceso de creación debe, por consiguiente, adoptar una estructura y régimen académico especialmente flexibles que le permita organizar rápidamente los cambios que llevan implícitos la creación e incorporación de nuevos conocimientos.

Asimismo, la diversidad de edades, capacidades, aspiraciones y ocupaciones de los estudiantes han impuesto cambios en la duración de las carreras, en las programaciones y en las estructuras curriculares (Neave, 2001). En este sentido, para el cumplimiento de este objetivo general la Misión Sucre se plantea objetivos específicos que apuntan hacia el desarrollo de currículos que impulsen el proyecto de Desarrollo Nacional endógeno y sostenible, así como también el desarrollo de un currículo abierto, flexible y dinámico que prevea la implementación de modalidades de estudios como la presencial, semi-presencial y la educación a distancia, entre otras (Ministerio de Educación Superior, 2003).

En esto coinciden autores como Neave (2001) e instituciones como la UNESCO (1998) al plantear que frente a los cambios sociales y el desarrollo científico, se hace necesario que la educación universitaria asuma modelos curriculares que estén adaptados a las exigencias contemporáneas de un mundo en permanente cambio y que responda a las expectativas personales de los estudiantes. Un modelo suficientemente flexible como para adaptarse a las condiciones actuales del país y que involucre al participante como un todo.

Por lo anteriormente expuesto, es importante analizar el cumplimiento de este objetivo desde la óptica de los actores involucrados. Analizar las respuestas de estudiantes, docentes y coordinadores abre la posibilidad de conocer el grado de coincidencia u oposición que sus opiniones tienen respecto a los supuestos que establece la Misión Sucre como política pública. Se emplea en este estudio el término Opinión como una creencia personal, un juicio o apreciación en relación con aspectos específicos de la ejecución de la Misión Sucre.

\section{A) Opiniones sobre las Modali-} dades de estudios

Con la Misión Sucre como política, no solamente se piensa en los jóvenes que, una vez concluidos sus estudios de 
Misión Sucre: Perfil estudiantil y opiniones de sus actores directos en Maracaibo Peña Ruiz, Rosa M.; Parra Olivares, Javier E. y Méndez de Souki, Betty

bachillerato, necesitan entrar en una opción universitaria, sino en la población que debe adaptarse a las cambiantes condiciones de trabajo, y para los cuales se han de preparar programas especiales, no carreras, para atender así en forma eficiente y con calidad escenarios diferentes de desarrollo y competencia. Es así como pensando en esa población se conciben varias modalidades de estudios tales como: la presencial, la semi-presencial, la educación a distancia y la no convencional ofreciéndoselas así los fines de semana y de lunes a viernes en horarios nocturnos. No obstante, hasta los momentos, la Misión Sucre sólo ha ofrecido la modalidad presencial, lo cual es considerado por la mayoría de los estudiantes en el caso de Maracaibo, como la opción que más le conviene con un $61,6 \%$; de ellos la mayoría $(85,6 \%)$ piensa que tiene mayor posibilidad de adquirir conocimientos con esa modalidad.

Pese a que la Misión Sucre se crea con el compromiso de formar nuevos ciudadanos, con oportunidades equitativas sin distinción de edad, género y situación socioeconómica, el hecho de sólo ofrecer la modalidad presencial a seis años de ejecución de la política, podría estar limitando el acceso a aquellas personas que no pueden estar permanentemente en un aula de clases. Siendo así, la misión estaría dejando de dar respuestas a una diversidad de demandantes que exigen cambios en los periodos y horarios.

Sin embargo, la preferencia por el tipo de modalidad presencial podría estar asociada a que $57,1 \%$ de la población estudiantil no trabaja y el $26 \%$ tiene un trabajo ocasional. Al cruzar la preferencia de la modalidad de estudio con condición la- boral se tiene que el $59,3 \%$ de quienes prefirieron la modalidad presencial no trabaja y un $24,2 \%$ tienen trabajo ocasional.

B) Opiniones sobre el Plan Curricular

En esta sección, se analizan las opiniones que tienen los actores que participan en la Misión Sucre (estudiantes, profesores, coordinadores) en relación al cumplimiento del objetivo que tiene que ver con el desarrollo de los currícula que contribuyan a impulsar el proyecto de desarrollo nacional endógeno y sostenible.

En la actualidad, una de las políticas y estrategias para el desarrollo de la educación universitaria en Venezuela, apunta hacia una mayor pertinencia social de las instituciones, los programas y los currícula. En este sentido, el término pertinencia ha sido utilizado para referirse a que la oferta educativa debe responder a las necesidades de los contextos.

Según Delgado (2004), unas de las formas de evidenciarse la pertinencia, es por medio de la coherencia que existe entre los objetivos y los perfiles terminales establecidos en los planes y programas de estudios. En correspondencia con lo planteado y tomando en cuenta el compromiso de la Misión Sucre, se describe a continuación la perspectiva que tienen los estudiantes en relación a dicho tema.

En el mejor de los casos la mayoría de los estudiantes opina que todas las asignaturas están acordes con las líneas estratégicas de la nación; en relación a la transformación con base al desarrollo endógeno el $60,3 \%$ opina que se cumple; el $50,8 \%$ de igual manera piensa que las unidades curriculares están acordes con las políticas del estado y la misma cifra, con la problemática de su municipio. 
Por otra parte, para conocer la opinión de los profesores sobre el cumplimiento de las directrices curriculares de la Misión Sucre, se les indicó que valoraran el nivel de cumplimiento de cada directriz como: alta, media o baja. Las opiniones sobre a) el nivel de cumplimiento en el que se están desarrollando estrategias de transformación con base al desarrollo endógeno, b) la adecuación de los contenidos de las unidades curriculares al plan de desarrollo nacional, c) la oferta de nuevas modalidades de estudio y d) la adecuación de la Misión Sucre a cada región, más de la mitad de los profesores manifestó que estas directrices se estaban cumpliendo a un nivel medio, mientras la directriz que tiene que ver con garantizar el acceso a la educación universitaria a todos, independientemente de su origen social, el $71,8 \%$ de los entrevistados manifestaron que se estaba cumpliendo a un nivel alto.

Por su parte los(as) coordinadores(as) de aldeas coinciden en todas sus opiniones acerca del cumplimiento de directrices. Todos opinan que los planes curriculares de las carreras que se imparten en la Misión Sucre contribuyen a impulsar el desarrollo endógeno y sostenible a un nivel alto. De igual manera, coinciden en que se cumple a un nivel alto tanto la adecuación de los contenidos de la materias al plan de desarrollo Nacional, así como también a las características de cada región. Por otra parte, el 100\% considera que se cumple a un nivel medio las estrategias de transformación, con base al desarrollo endógeno y el acceso a la Educación universitaria a todos, independientemente de su origen social. Respecto a la diversidad de modalidades de es- tudios que ofrece la Misión Sucre, el $100 \%$ considera que se cumple a un nivel muy bajo.

El $57,1 \%$ de los(as) coordinadores(as) señala que la promoción e implantación de modelos educativos se está haciendo a un nivel medio, mientras que el $100 \%$ de los entrevistados opina que no se está conformando la red que integra a todas las instituciones que cooperan con la Misión Sucre y que los currículos existentes no contribuyen a impulsar el proyecto de desarrollo nacional endógeno y sostenible. Por otra parte el $71,4 \%$ considera que se han propiciado a un nivel alto currículos flexibles, abiertos, dinámicos e innovadores.

C) Opiniones sobre calidad de los servicios estudiantiles y procesos de aprendizaje

Este aspecto fue explorado a través del cuestionario solicitándoles a los(as) estudiantes que valoraran las condiciones de las aldeas como muy buena, buena, regular, mala, muy mala o no existe.

Los resultados arrojaron que el $48,2 \%$ considera las condiciones de las aulas como buenas o muy buenas, mientras el $32,7 \%$ opina que las condiciones de las aulas son regulares. Un 31,4\% de igual manera percibe como regulares las condiciones de los mobiliarios, carecen de servicios de reproducción y biblioteca en las aldeas y algunas de éstas cuentan con servicios de cafetería.

Con relación a las relaciones con el coordinador de su aldea, el $59 \%$ opina que hay buena o muy buena receptividad de su parte. Por otro lado al preguntarle sobre la calidad de la capacidad didáctica de los profesores, el 95,2\% opina que tiene buena o muy buena capacidad didáctica, de 
Misión Sucre: Perfil estudiantil y opiniones de sus actores directos en Maracaibo Peña Ruiz, Rosa M.; Parra Olivares, Javier E. y Méndez de Souki, Betty

igual manera un $90,5 \%$ opina que son buenos o muy buenos los métodos de evaluación aplicados por los docentes.

Por otra parte, pese a que muchas aldeas universitarias se encuentran en malas condiciones de infraestructura y carecen de los recursos mínimos tales como: bibliotecas y servicios de reproducción, el $71,1 \%$ de los estudiantes manifiesta que recomendarían a otros estudiar en la Misión Sucre, ya que la ven como la única oportunidad de lograr una carrera universitaria a pesar de todas sus deficiencias.

Así mismo, en cuanto a la pregunta ¿bajo qué condiciones dejarías de estudiar en la Misión Sucre?, el 47,3\% respondió que bajo ninguna condición, seguido de un porcentaje muy lejano que mencionó como causa alguna enfermedad. Estas cifras indican el grado de identificación de los beneficiarios con esta política.

En cuanto a los profesores-asesores, la mayoría considera que la situación de trabajo fuera de los salones de clase es regular $(64,1 \%)$, las mayores insatisfacciones se detectan en el acceso a Internet (23\% lo califica como insuficiente) y las bibliotecas (25,65\% sostiene que no existen). Esto podría desmotivar la acción del docente ya que se desenvuelve en un ambiente incómodo, estrecho e inadecuado, influyendo no solamente en su desempeño laboral sino también en la calidad del proceso de enseñanza que él o ella facilita.

D) Sinergia Institucional para el desarrollo de la Misión Sucre

La Fundación Misión Sucre tiene como una de sus estrategias, la articulación con otras instituciones que coadyuven al logro de los objetivos, en el marco de la sinergia y concurrencia que se requieren en la nueva institucionalización para avanzar en la conformación de la nueva estructura social. De igual manera, tiene la responsabilidad y el compromiso de articularse con otras instituciones y organismos que, por la naturaleza de sus propósitos, se orientan también a la inclusión social.

Teniendo en cuenta que la Misión Sucre, para resolver el problema de la exclusión estudiantil a nivel universitario, ha planteado la necesidad de trabajar articuladamente para desarrollar las actividades necesarias en el logro de los objetivos planeados, se hace necesario revisar hasta que punto se ha logrado esa sinergia institucional en el Municipio Maracaibo. Para la descripción de este aspecto se sistematizó la información suministrada por la coordinación estadal y los coordinadores de las aldeas, a partir de una entrevista semi-estructurada.

A nivel regional instituciones como Corpozulia, la Fuerza Armada, Pdvsa, Hidrolago y la Zona Educativa han participado en la ejecución de la Misión Sucre, desarrollando o apoyando algunas acciones de manera puntual. En el caso de la Zona Educativa, el apoyo está relacionado con la utilización de las sedes de algunas escuelas como aldeas universitarias en horario nocturno y los fines de semana.

En el Municipio Maracaibo, se ha avanzado hacia el establecimiento de compromisos de las instituciones de educación universitaria, a través de las distintas coordinaciones y su seguimiento, así como en la generación de un espacio de trabajo por sub-regiones en función del monitoreo y control de la Misión Sucre. 
Las principales dificultades encontradas, según opinión de los coordinadores de las aldeas, hacen referencia a la magnitud del problema frente a las capacidades institucionales, evidenciándose una débil coordinación tanto inter como intra-institucional. Los problemas organizacionales al interior de las instituciones fragmentan las acciones e impiden el accionar conjunto hacia el cumplimiento del propósito de la política. Otra dificultad encontrada ha sido la alta movilidad de los funcionarios que se encuentran al frente de los programas.

\section{Consideraciones finales}

Según el documento "Fundamentos Conceptuales de la Misión Sucre (Ministerio de Educación Superior, 2003) una de las razones principales por las cuales nace esta política es para garantizar la inclusión a este subsistema de educación a bachilleres que no pudieron cursar estudio en las instituciones de educación universitaria, proponiendo un conjunto de objetivos sociales e institucionales que orientan hacia la transformación de fondo de la educación universitaria.

Tomando en cuenta los resultados obtenidos a lo largo del texto en relación al cuestionario aplicado a una muestra de estudiantes de la Misión Sucre en Maracaibo, se encontró que el $62,5 \%$ de los estudiantes pertenecen al estrato IV (pobreza relativa), el $75,9 \%$ provienen de liceos públicos y el $67 \%$ son del género femenino. En términos de edad, se encontró que el $28,9 \%$ de los estudiantes se encuentra ubicado en los grupos de edades de más de 40 años, datos que demuestran que esta misión ha contribuido a in- corporar a la educación universitaria a aquellos estudiantes que fueron excluidos en años anteriores y que además provienen de los estratos socioeconómicos menos favorecidos.

Por otro lado, se pudo constatar que aunque la Misión Sucre establece varias modalidades de estudio tales como: la presencial, la semi-presencial, la educación a distancia y la no convencional, hasta los momentos, sólo se le ha ofrecido la modalidad presencial a los estudiantes que residen en Maracaibo, no cumpliendo así la Misión Sucre, con el objetivo especifico que tiene que ver con la implementación de diferentes modalidades de formación. No obstante, vale la pena resaltar que el hecho de que sólo se ofrezca la modalidad presencial, los estudiantes no lo ven como negativo, pues la mayoría opina que esa es la opción que más le conviene porque tienen mayor posibilidad de adquirir conocimientos.

Resulta importante resaltar que los estudiantes en Maracaibo, pese a que consideran que las condiciones de las aldeas en relación a dotación y a infraestructura son deficientes, ven a la Misión Sucre, como una oportunidad única de lograr una carrera universitaria y que bajo ninguna circunstancia dejarían de estudiar en ella, lo que demuestra un alto grado de sentido de pertenencia o identificación de los beneficiarios con esta política.

A pesar de la posición de los docentes que declaran las precarias condiciones de trabajo que existen en todas las aldeas, en cuanto a: acceso a Internet, bibliotecas y equipos audiovisuales, habría que enfatizar que en Maracaibo hay una proporción bastante alta de docentes que opina que la Misión Sucre está cumplien- 
Misión Sucre: Perfil estudiantil y opiniones de sus actores directos en Maracaibo Peña Ruiz, Rosa M.; Parra Olivares, Javier E. y Méndez de Souki, Betty

do en un nivel alto los objetivos que tienen que ver con garantizar el acceso a la educación universitaria y la adecuación de los planes curriculares al plan de desarroIlo nacional.

Ante el esfuerzo de la Misión Sucre de lograr una sinergia institucional donde se conformen y vinculen todas aquellas instituciones y factores vinculados a la educación universitaria para propiciar una verdadera transformación de la educación universitaria, parecen insuficientes o parciales los mecanismos que hasta la actualidad se han llevado a cabo para conformar esa red, porque a pesar de los intentos que se han realizado en cuanto a una planificación participativa pareciera que no se ha logrado integrar al conjunto de las Instituciones de Educación Universitaria con otras instituciones del Estado para la ejecución de la Misión Sucre en Maracaibo. La participación de las diferentes instituciones educativas que funcionan en el municipio Maracaibo se han visto poco reflejada como lo evidencia el presente trabajo.

\section{Referencias Bibliográficas y Documentales}

Asamblea Nacional (2000). Constitución de la República Bolivariana de Venezuela, Segunda versión. Gaceta Oficial 5.453 del 24 de Marzo.

Alvarez, Ángel (1992). Análisis de Políticas públicas. Centro de documentación y análisis de información del Centro Latinoamericano de Administración (CLAD).

Alvarez, Ángel (1994). Modernización de la Gestión Pública y del Proceso de Formación de Políticas Públicas en el marco de la Reforma del Estado. En: FERNÁNDEZ, Julio César y ME-
DINA, Antonio (coord.) "Reformas de las Instituciones de Gobierno (Propuesta para la modernización del poder Ejecutivo)", COPRE, volumen 11, Caracas, Venezuela.

CORPOZULIA-FUNDACREDESA (2001). Estudio Condiciones de vida de la población zuliana. Edición institucional, Maracaibo, Venezuela.

Delgado, Juan C. (2004). La transformación Universitaria. Como respuesta a los cambios de la contemporaneidad. Universidad de los Andes, Consejo de publicaciones. Mérida, Venezuela.

Fuenmayor, Luis y Vidal, Yasmila (2000). Unidad de Estadística CNU-OPSU, Ediciones OPSU, Caracas, Venezuela.

Fuenmayor, Luis (2002). "A propósito de las iniquidades en el Ingreso a la Educación Superior". Revista Venezolana de Gerencia. Facultad de Ciencias Económicas y Sociales, LUZ. Año 7, No. 17, Maracaibo, Venezuela.

Fuenmayor, Luis (2003). Comparación del perfil de los diez mejores aspirantes a ingresar en las instituciones de educación superior venezolanas en los períodos 2002-2003 y 2003-2004. Revista Venezolana de Educación Superior. CNU-OPSU, Vol. 1. No. 1.Ediciones OPSU, Caracas, Venezuela.

Fundación Misión Sucre (2003). Censo para la Misión Sucre. Caracas, Venezuela.

Fundación Misión Sucre (2009). Sistema de Información Nacional de Educación Superior (Reporte), Caracas, Venezuela.

Ministerio de Educación Cultura y Deportes (2001). Políticas y estrategias para el desarrollo de la Educación Superior en Venezuela 2000-2006. Caracas, Venezuela.

Ministerio de Educación Superior (2003). Fundamentos Conceptuales de la Misión Sucre, Caracas, Venezuela. 
Morales Gil, Eduardo (2003). La Exclusión de los Pobres de la Educación Universitaria Venezolana. Ediciones OPSU, Caracas, Venezuela.

Neave, Guy (2001). Educación Superior: Historia y política. Estudios comparativos sobre la universidad contemporánea, Gedisa Editorial, Barcelona, España.

OPSU (2002). Estadísticas de la educación superior. Caracas, Venezuela.

Parra Olivares, Javier (2006). Guía de Muestreo. Tercera edición. Colección XLV aniversario FCES. Maracaibo, Venezuela.

Sarco Lira, Amalio y Bonucci, Mario (1998). La Política de Admisión en Venezuela. Ponencia al II Encuentro de Secretarios de las Universidades de América Latina y el Caribe. La Habana, Cuba.

Subirats, Joan (1996). Análisis de Políticas Públicas y Gestión Pública. Promesas y riesgos de una vida en común en: Lecturas de Gestión Pública, Instituto Nacional de Administración Pública, Madrid, España.

Schneckenberg, Marisa (2003). La implementación del PROEM (Programa Expansión, Mejora e Innovación en la Enseñanza Media) como política educativa en la práctica de la gestión escolar. IIPE-UNESCO, Buenos Aires, Argentina.

UNESCO (1998). Declaración Mundial sobre la Educación Superior en el siglo XXI: Visión y Acción. En Conferencia Mundial sobre la Educación Superior. http://www.unesco.org/education/educprog/wche/declaration spa.htm (consultada el 20-05-09)

Vallés, Joseph (2000). Ciencia Política: una introducción. Editorial Ariel, Barcelona, España. 